\title{
MHD modelling of coronal loops: injection of high-speed chromospheric flows ${ }^{\star}$
}

\author{
A. Petralia ${ }^{1}$, F. Reale ${ }^{1,2}$, S. Orlando ${ }^{2}$, and J. A. Klimchuk ${ }^{3}$ \\ 1 Dipartimento di Fisica \& Chimica, Università di Palermo, Piazza del Parlamento 1, 90134 Palermo, Italy \\ e-mail: apetralia@astropa.unipa.it \\ 2 INAF - Osservatorio Astronomico di Palermo, Piazza del Parlamento 1, 90134 Palermo, Italy \\ 3 NASA Goddard Space Flight Center, Greenbelt MD 20771, USA
}

Received 8 November 2013 / Accepted 29 April 2014

\begin{abstract}
Context. Observations reveal a correspondence between chromospheric type II spicules and bright upward-moving fronts in the corona observed in the extreme-ultraviolet (EUV) band. However, theoretical considerations suggest that these flows are probably not the main source of heating in coronal magnetic loops.

Aims. We investigate the propagation of high-speed chromospheric flows into coronal magnetic flux tubes and the possible production of emission in the EUV band.

Methods. We simulated the propagation of a dense $10^{4} \mathrm{~K}$ chromospheric jet upward along a coronal loop by means of a 2D cylindrical MHD model that includes gravity, radiative losses, thermal conduction, and magnetic induction. The jet propagates in a complete atmosphere including the chromosphere and a tenuous cool $(\sim 0.8 \mathrm{MK})$ corona, linked through a steep transition region. In our reference model, the jet initial speed is $70 \mathrm{~km} \mathrm{~s}^{-1}$, its initial density is $10^{11} \mathrm{~cm}^{-3}$, and the ambient uniform magnetic field is $10 \mathrm{G}$. We also explored other values of jet speed and density in $1 \mathrm{D}$ and different magnetic field values in $2 \mathrm{D}$, as well as the jet propagation in a hotter ( 1.5 MK) background loop.

Results. While the initial speed of the jet does not allow it to reach the loop apex, a hot shock-front develops ahead of it and travels to the other extreme of the loop. The shock front compresses the coronal plasma and heats it to about $10^{6} \mathrm{~K}$. As a result, a bright moving front becomes visible in the $171 \AA$ channel of the SDO/AIA mission. This result generally applies to all the other explored cases, except for the propagation in the hotter loop.

Conclusions. For a cool, low-density initial coronal loop, the post-shock plasma ahead of upward chromospheric flows might explain at least part of the observed correspondence between type II spicules and EUV emission excess.
\end{abstract}

Key words. Sun: chromosphere - Sun: corona - Sun: UV radiation - magnetohydrodynamics (MHD)

\section{Introduction}

Type II spicules are very dynamic chromospheric events, with lifetimes ranging from 10 to $150 \mathrm{~s}$ and velocities between 50 and $150 \mathrm{~km} \mathrm{~s}^{-1}$ (De Pontieu et al. 2007). Cold plasma flows upward at $10^{4} \mathrm{~K}$, and it has been proposed that a fraction of the plasma is heated to coronal temperatures (De Pontieu et al. 2011; Madjarska et al. 2011; Vanninathan et al. 2012). For this reason and because they are very frequent events, it has been proposed that both ordinary spicules and type II spicules contribute substantially to sustain the high coronal temperatures (Beckers 1978; Pneuman \& Kopp 1978; Athay \& Holzer 1982; Tsiropoula \& Tziotziou 2004; De Pontieu et al. 2009).

Klimchuk (2012) has discussed three observational tests of the hypothesis that most coronal plasma originates in the hot tips of spicules. These tests involved the ratios of the blue wing to the line core intensity, ratios of the lower transition region to the coronal emission measure, and the ratio of the blue wing

\footnotetext{
* Movies associated to Figs. 3, 6, 7 are available in electronic form at http://www. aanda.org
}

to the line core density. The hypothesis failed all three tests by a wide margin (Klimchuk 2012; Tripathi \& Klimchuk 2013; Patsourakos et al. 2014). The implication is that type II spicules inject at most a small fraction of the hot plasma in the corona. Nonetheless, the correspondence between type II spicules and coronal extreme-ultraviolet (EUV) emission is well supported. It is therefore very important to understand how these flows are able to produce the observed emission.

One possible scenario is that these jets produce shocks that propagate inside the loop and heat and compress the plasma that is already there. This might produce a transient excess EUV emission that would be observed. That chromospheric material injected into a coronal flux tube produces a shock that can heat the coronal plasma has been known for some time (e.g., Karpen et al. 1982). Here we focus on the shock-heated material in front of the chromospheric jet.

We explore this scenario with a detailed magnetohydrodynamical (MHD) model and test it quantitatively. A dense flow is triggered at one end of a magnetic flux tube that links two chromospheres through a tenuous corona. We study the 
propagation of this flow and investigate whether it drives signatures in EUV observations. In Sect. 2 the model is described, we report on the simulations and the results in Sect. 3, and discuss them in Sect. 4.

\section{Model}

We modelled the propagation of a dense chromospheric jet into a closed coronal loop that is anchored to the solar chromosphere. We simplified the model geometry to that of a straight magnetic flux tube that links two independent chromospheres. We imaged it as a vertical flux tube in 2D cylindrical geometry, that is, the magnetic field is in the vertical direction. However, we maintained the gravity of a semicircular closed loop, that is, the gravity is reduced as we move farther from the chromosphere and is zero at the loop apex, which corresponds to half the distance between the boundaries at the middle of the vertical axis.

As our simulation strategy, we considered a reference model and then a number of other case studies with deviations from the reference model by one or more parameters. Throughout all the modelling, the initial condition was a complete coronal loop atmosphere. To test the suggestion that the corona comes from type II spicules, our reference loop atmosphere consisted of an initially very tenuous corona (EUV-dark) linked to a thin chromospheric layer through the usual steep transition region. To understand the hot emission from type II spicules, even if it does not explain the bulk of the corona, we ran a simulation that used typical conditions of an EUV-bright corona. The atmosphere was immersed in a uniform magnetic field that links the two chromospheres. In the reference case, the intensity of the magnetic field implies values of the plasma $\beta \leq 1$ (see below), which means that the plasma is mostly confined by the field. For completeness, we also considered a case of a lower magnetic field and plasma $\beta \geq 1$.

In this atmosphere, a jet was injected from the bottom boundary upward at time $t=0$. Initially, the upflow therefore had chromospheric density and temperature. We wished to study the propagation of the shock front that develops ahead of the upflow along the loop, and desired to maximize the observable effects from warm plasma emitting in the EUV band. Therefore we did not let the cold chromospheric material fill the loop completely. To do this, the speed was chosen to be relatively high to produce a shock front, but not so high as to let the flow reach (and overcome) the loop apex. The resulting velocities of both the cold and the hot plasma are fully consistent with observations.

Our model solves the MHD equations for an ideal compressible plasma in the following conservative form:

$$
\begin{aligned}
& \frac{\partial \rho}{\partial t}+\nabla \cdot(\rho \boldsymbol{v})=0 \\
& \frac{\partial \rho \boldsymbol{v}}{\partial t}+\nabla \cdot\left(\rho \boldsymbol{v} \boldsymbol{v}-\boldsymbol{B B}+p_{t} \boldsymbol{I}\right)=\rho \boldsymbol{g} \\
& \frac{\partial E}{\partial t}+\nabla \cdot\left(\left(E+p_{t}\right) \boldsymbol{v}-\boldsymbol{B}(\boldsymbol{v} \cdot \boldsymbol{B})\right)=\rho \boldsymbol{v} \cdot \boldsymbol{g}-n_{e} n_{\mathrm{H}} \Lambda(T)+H-\nabla \cdot \boldsymbol{F}_{c} \\
& \frac{\partial \boldsymbol{B}}{\partial t}+\nabla \cdot(\boldsymbol{v} \boldsymbol{B}-\boldsymbol{B} \boldsymbol{v})=0 \\
& \nabla \cdot \boldsymbol{B}=0
\end{aligned}
$$

where

$\rho=\mu \mathrm{m}_{\mathrm{H}} n_{\mathrm{H}}$

$p_{t}=p+\frac{\boldsymbol{B} \cdot \boldsymbol{B}}{2}$

$E=\rho \epsilon+\rho \frac{\boldsymbol{v} \cdot \boldsymbol{v}}{2}+\frac{\boldsymbol{B} \cdot \boldsymbol{B}}{2}$

$\boldsymbol{F}_{c}=\frac{F_{\text {sat }}}{F_{\text {sat }}+\left|\boldsymbol{F}_{\text {class }}\right|} \boldsymbol{F}_{\text {class }}$

$\boldsymbol{F}_{\text {class }}=k_{\|} \boldsymbol{b}(\boldsymbol{b} \cdot \nabla T)+k_{\perp}(\nabla T-\boldsymbol{b}(\boldsymbol{b} \cdot \nabla T))$

$\left|\boldsymbol{F}_{\text {class }}\right|=\sqrt{(\boldsymbol{b} \cdot \nabla T)^{2}\left(k_{\|}^{2}-k_{\perp}^{2}\right)+k_{\perp}^{2} \nabla T^{2}}$

$F_{\text {sat }}=5 \Phi \rho c_{\mathrm{s}}^{3}$,

where $\mu=1.265$ is the mean atomic mass (assuming solar metal abundances; Anders \& Grevesse 1989), $m_{\mathrm{H}}$ is the mass of the hydrogen atom, $n_{H}$ is the hydrogen number density, $p_{t}$ is the total pressure, that is, the sum of the thermal pressure and the magnetic pressure (the factor $1 / \sqrt{4 \pi}$ is absorbed in the definition of $\boldsymbol{B}), E$ is the total energy density, that is, the sum of the thermal energy density $(\rho \epsilon)$, the kinetic energy density and the magnetic energy, $\boldsymbol{v}$ is the plasma velocity, $\boldsymbol{g}$ is the solar gravity, $\Lambda(T)$ is the radiative loss function for optically thin plasma, $\boldsymbol{F}_{c}$ is the conductive flux, $H$ is a heating function whose only role is to keep the unperturbed atmosphere in energy equilibrium, $c_{\mathrm{S}}$ is the sound speed for an isothermal plasma, $\Phi$ is a free parameter $(<1$, Giuliani 1984) that determines the degree of saturation of the thermal conduction; we set $\Phi=0.9$, which corresponds to quite an efficient conduction. The radiative losses were computed according to version 7 of the CHIANTI code (Landi et al. 2012), assuming a density of $10^{9} \mathrm{~cm}^{-3}$ and ionization equilibrium according to Dere (2009). We did not account for the radiative losses of the chromospheric plasma, therefore we set $\Lambda(T)=0$, as well as $H=0$, for $T<10^{4} \mathrm{~K}$.

We completed this set with the equation of state for an ideal gas:

$p=(\gamma-1) \rho \epsilon$.

The calculations were performed using the PLUTO code (Mignone et al. 2007, 2012), a modular, Godunov-type code for astrophysical plasmas. The code provides a multiphysics, algorithmic modular environment particularly oriented toward the treatment of astrophysical flows in the presence of discontinuities, as in the case treated here. The code was designed to make efficient use of massive parallel computers using the messagepassing interface (MPI) library for interprocessor communications. The MHD equations were solved using the MHD module available in PLUTO, configured to compute intercell fluxes with the Harten-Lax-Van Leer approximate Riemann solver, while second-order in time was achieved using a Runge-Kutta scheme. A Van Leer limiter for the primitive variables was used. The evolution of the magnetic field was carried out by adopting the constrained transport approach (Balsara \& Spicer 1999) that maintains the solenoidal condition $(\nabla \cdot B=0)$ at machine accuracy. PLUTO includes optically thin radiative losses in a fractional step formalism (Mignone et al. 2007), which preserves the second-order time accuracy because the advection and source steps are at least accurate to second order; the radiative losses $\Lambda(T)$ values were computed at the temperature of interest using a table lookup/interpolation method. The thermal conduction was treated separately from advection terms through operator splitting. In particular, we adopted the super-time-stepping technique (Alexiades et al. 1996), which has been proved to be 


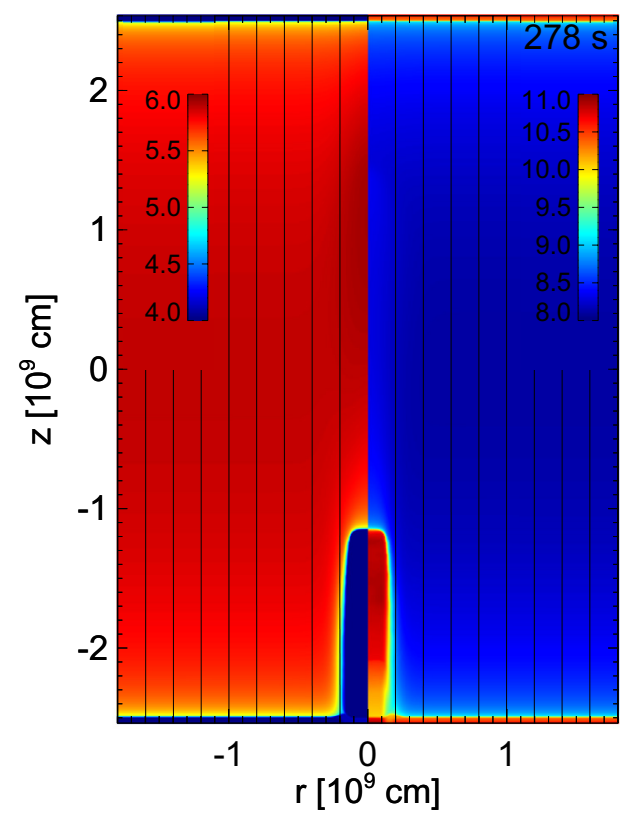

(a)

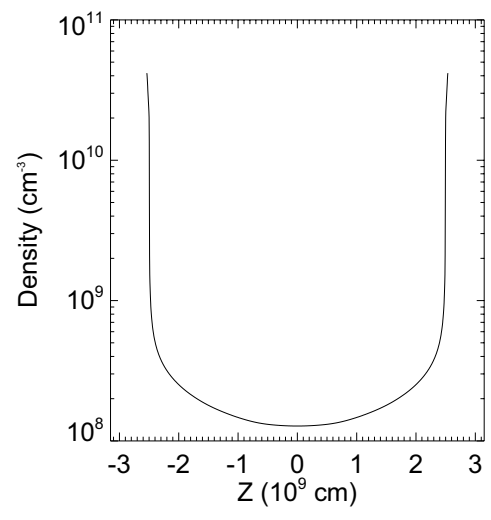

(b)

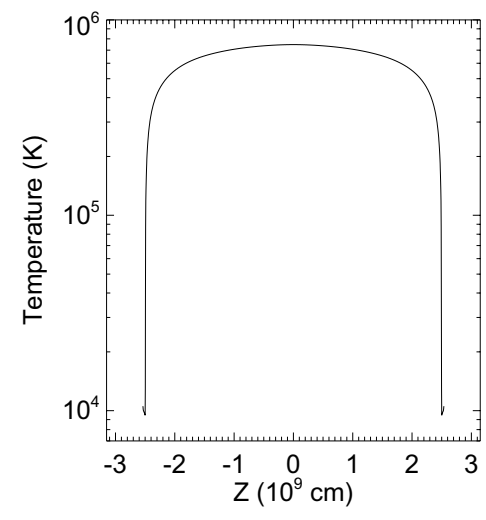

Fig. 1. Configuration of the computational domain: a) maps of density $\left(r i g h t, \mathrm{~cm}^{-3}\right)$ and temperature $(l e f t, \mathrm{~K})$, in logarithmic scale, at time $t=278 \mathrm{~s}$; b) unperturbed density and temperature along the $z$ axis.

very effective to speed up explicit time-stepping schemes for parabolic problems. This approach is crucial when high values of plasma temperature are reached (as during flares). In fact, the explicit scheme would require a very small time step because of the very restrictive stability condition, which is $\Delta t \leqslant(\Delta x)^{2} / \eta$, where $\eta$ is the maximum diffusion coefficient (e.g., Orlando et al. 2005, 2008).

\subsection{Loop setup}

In the reference model, the computational domain is twodimensional and cylindrical $(r, z)$, and extends over $2 L \sim 5.08 \times$ $10^{9} \mathrm{~cm}$ in the $z$ direction and $R_{L} \sim 1.8 \times 10^{9} \mathrm{~cm}$ in the $r$ direction, as shown in Fig. 1a. As reference initial conditions, we considered a hydrostatic and relatively tenuous and cool unperturbed loop atmosphere, (e.g. Reale 2010); the density and temperature were set to be $n \sim 5 \times 10^{8} \mathrm{~cm}^{-3}$ and $T \sim 8 \times 10^{5} \mathrm{~K}$, respectively, at the loop apex, that is, at $z=0$, and $n \sim 10^{11} \mathrm{~cm}^{-3}$ and $T \sim 10^{4} \mathrm{~K}$ at the base of the chromosphere, with the profiles shown in Fig. 1b. The coronal heating is uniform with a value $H \sim 4.2 \times 10^{-5} \mathrm{erg} \mathrm{cm}^{-3} \mathrm{~s}^{-1}$. As alternative conditions, we also considered an initially hotter loop, where the density and temperature are $n \sim 10^{9} \mathrm{~cm}^{-3}$ and $T \sim 1.5 \times 10^{6} \mathrm{~K}$, respectively, at the apex and a denser chromosphere where $n \sim 10^{12} \mathrm{~cm}^{-3}$ and $T \sim 10^{4} \mathrm{~K}$ at base of the chromosphere, with a uniform coronal heating of $H \sim 4.68 \times 10^{-4} \mathrm{erg} \mathrm{cm}^{-3} \mathrm{~s}^{-1}$.

The magnetic field is uniform in the $z$ direction and, in our reference configuration, had an intensity of $10 \mathrm{G}$. The field is therefore able to confine the plasma in the corona because the plasma parameter $\beta$ is estimated to be

$\beta=\frac{p_{\text {thermal }}}{p_{\text {magnetic }}}=\frac{2 n K_{B} T}{B^{2} / 8 \pi} \sim 0.1$.

Alternatively, we also considered a magnetic field with an intensity of $3 \mathrm{G}$, that, together with a higher density of the jet, leads to a higher value of $\beta \geq 1$. We are aware that for a low value of $\beta$ the plasma evolution occurs mostly along the magnetic field lines, nevertheless, for our reference model we equally considered a fully 2D MHD description. This allowed us to compare this directly with the case at higher $\beta$, which involves deviations from confinement. For the other simulations, the $2 \mathrm{D}$ description is unnecessary, and we considered a more efficient $1 \mathrm{D}$ geometry.

We set axisymmetric conditions at $r=0$ for the boundary conditions and reflective conditions at $r=R_{L}$, where the magnetic field maintains the parallel component and reverses the perpendicular component (to the $z$ direction). At the $z$ boundaries we assumed reflective conditions and the magnetic field maintains the perpendicular component and reverses the parallel component, except where the jet is defined. There we assumed inflow boundary conditions.

The mesh of the $2 \mathrm{D}$ domain is $z \times r=1710 \times 160$ grid points and is uniformly spaced all along the $z$-axis $\left(\mathrm{d} z=3.0 \times 10^{6} \mathrm{~cm}\right)$, and partially along the $r$-axis, from the center $r=0$ to a distance $r=R_{U}=1.04 \times 10^{9} \mathrm{~cm}\left(\mathrm{~d} r=8.2 \times 10^{6} \mathrm{~cm}\right)$. For $r>R_{U}$ the spacing gradually expands to $\mathrm{d} r=5.2 \times 10^{7} \mathrm{~cm}$; thus the boundary conditions are far enough to avoid any effect on the jet region. The uniformly high resolution along the $z$ direction allows us to accurately describe both the steep transition region and the moving shock front. To determine that the resolution in the steep gradient regions was high enough (e.g., Bradshaw \& Cargill 2013), we made a test at higher spatial resolution along $z\left(\mathrm{~d} z=0.5 \times 10^{6} \mathrm{~cm}\right)$ and ascertained that the results do not change except for a few details.

\section{Simulation}

In the reference simulation, the jet was injected at the lower boundary of the domain with a velocity of $70 \mathrm{~km} \mathrm{~s}^{-1}$ within a distance of $\delta r=1.5 \times 10^{8} \mathrm{~cm}$ from the $z$ axis. We made some other tests with different jet velocities, 30,50 and $90 \mathrm{~km} \mathrm{~s}^{-1}$. Because spicules are observed to have a lifetime of a few minutes at most, for all simulations the flow was assumed to have a finite duration, described by the time profile in Fig. 2. The bulk 


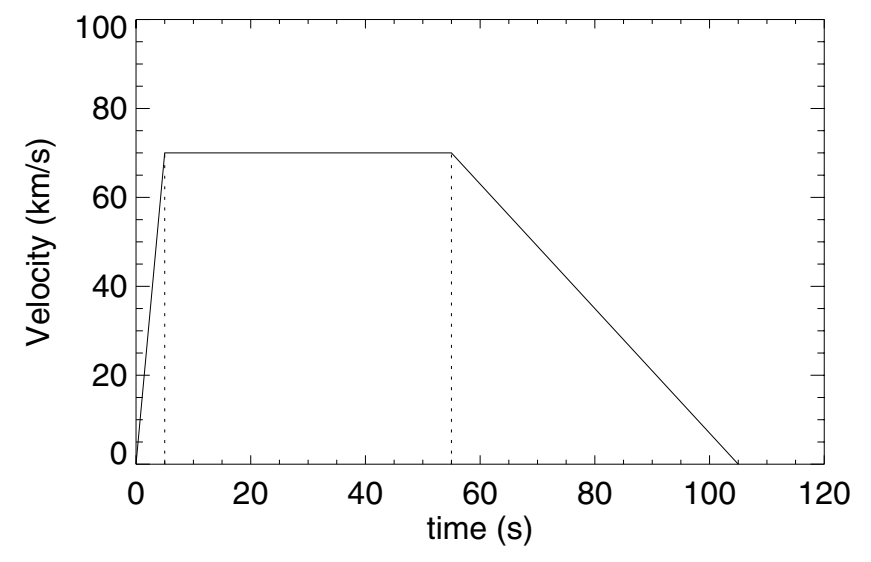

Fig. 2. Temporal profile of the cold jet.

of the injection lasts $50 \mathrm{~s}$. We set a linear rise and decrease of the flow speed that avoided numerical problems due to boundary conditions. The total duration of the flow was about $2 \mathrm{~min}$. We do not expect significant differences of the relevant results for different flow durations in the observed range.

Because the confinement of the plasma is efficient, the thickness of the flow defines the thickness of the loop in which the jet propagates in the reference case (Fig. 1a). The flow is defined solely by the velocity at the lower boundary of the domain. The density and temperature are the same as those of the medium from which it originates, that is, $n \sim 10^{11} \mathrm{~cm}^{-3}$ and $T \sim 10^{4} \mathrm{~K}$. We did not account for possible loop expansion from the chromosphere to the corona (Guarrasi et al. 2014).

The evolution of the simulated jet is presented in Figs. 3 and 4. This work addresses the evolution of the coronal plasma outside of the jet, therefore we focused on the dynamics of the jet and do not discuss the jet internal structure and its thermal evolution in detail. The speed value does not allow the jet to reach the apex of the loop, therefore it returns to the chromosphere because of gravity after travelling a distance of $\sim 2 \times 10^{9} \mathrm{~cm}$ (from the base of the loop) in $500 \mathrm{~s}$. This distance corresponds to an altitude of $\sim 1.6 \times 10^{9} \mathrm{~cm}$ with our curved-loop gravity function, and is consistent with a simple ballistic estimate,

$h=\frac{v^{2}}{2 g_{\odot}} \sim 10^{9} \mathrm{~cm}$.

Figure 3 shows maps of temperature and density through a cross-section of the cylindrical domain at four subsequent times (Fig. 1a shows the last frame of Figs. 3 with a different, broader colour scale).

At $t=69 \mathrm{~s}$, the cold jet has overcome the chromosphere, having travelled for a distance $\Delta z \sim 5 \times 10^{8} \mathrm{~cm}$, and it is clearly visible as a blue bump in the temperature map. A shock front also propagates ahead of the cold temperature front and is visible as a red bump in the density map. A weaker thermal front, due to the efficient thermal conduction, is visible ahead of the shock as a slight reddening along the central axis in the temperature map. At time $t=139 \mathrm{~s}$, the jet is no longer being driven at the base. The fronts have all moved upward, with different speeds that enlarge the distances between them. While the jet has advanced only to a height of $\sim 10^{9} \mathrm{~cm}$, the shock has almost reached the loop apex and the thermal front has a long tail that extends even farther. At $t=208 \mathrm{~s}$, the jet has reached its maximum height and is now almost stationary because of the gravitational deceleration. The shock and the thermal front are instead still moving ahead to the other end of the loop. At $t=278 \mathrm{~s}$, the thermal front has reached the other end of the loop, preceding the shock. As mentioned above, the magnetic field is only weakly affected by the propagation of the jet, which remains well collimated and confined, with a sharp flat front, as shown in Fig. 1a. Some weak density and temperature halo is visible at the base of the corona in the lower panels of Fig. 3, emphasized by the saturation from the expanded logarithmic scale. The post-shock plasma has some small expansion while linking to the cold jet at the back. The round shape of the jet front in Fig. 3 is only apparent because of the saturated colour scale (see Fig. 1a).

Later the jet stops and eventually returns to the surface, while the shock hits the chromosphere at the other end of the loop. This later evolution (not shown) does not add interesting information to our discussion.

We now focus on some quantitative details. Figure 4 shows profiles of temperature, density (logarithmic scale), and velocity along the central axis of the domain.

The density peak $\left(n \sim 2 \times 10^{11} \mathrm{~cm}^{-3}\right)$ marks the head of the cold jet that moves to the right in the plot. At $t=69 \mathrm{~s}$ its front is at $z \sim-2 \times 10^{9} \mathrm{~cm}$ and has moved to $z \sim-10^{9} \mathrm{~cm}$ at $t=278 \mathrm{~s}$, with an average speed of $\sim 50 \mathrm{~km} \mathrm{~s}^{-1}$. Due to the large heat capacity of the jet, the hotter corona above is unable to heat the cold jet plasma by thermal conduction and the jet temperature remains mostly below $10^{4} \mathrm{~K}$ throughout its propagation. The density of the jet decreases gradually below $10^{11} \mathrm{~cm}^{-3}$ with time. The plasma slows down from $\sim 60 \mathrm{~km} \mathrm{~s}^{-1}$ to $\sim 30 \mathrm{~km} \mathrm{~s}^{-1}$ (it will stop completely later on and return to the chromosphere).

At $t=69 \mathrm{~s}$ on the left side of the temperature profile $\left(z \approx-1.6 \times 10^{9} \mathrm{~cm}\right)$ we see a small cusp at about $10^{6} \mathrm{~K}$. This cusp marks the shock front, which is more clearly visible as a jump in the density profile and as a sharp velocity peak (at $80 \mathrm{~km} \mathrm{~s}^{-1}$ ). This front propagates to the right along $z$ with an approximately constant speed $v_{\mathrm{sh}} \sim 140 \mathrm{~km} \mathrm{~s}^{-1}$. At $t=278 \mathrm{~s}$ the shock front is nearly at the right end of the domain $\left(z \sim 1.5 \times 10^{9} \mathrm{~cm}\right)$. The density jump is somewhat reduced during the propagation and the plasma speed at the shock front also decreases to about $60 \mathrm{~km} \mathrm{~s}^{-1}$.

A detail of this evolution at time $t=139 \mathrm{~s}$ is shown in Fig. 5 . The shock front is very well visible in all three plots. At the sides of the temperature cusp the temperature is smoothly linked to the original temperature profile. This smooth trend is the thermal front that we see in Fig. 3. The jump in density between preand post-shock medium is approximately a factor of two. This scenario is consistent with the estimates described below.

Since the efficient thermal conduction in the corona makes the shock nearly isothermal, the isothermal sound speed is the reference speed:

$c_{\mathrm{s}} \approx \sqrt{\frac{2 k_{\mathrm{B}} T_{\mathrm{sh}}}{\mu \mathrm{m}_{\mathrm{H}}}} \sim 100 \mathrm{~km} \mathrm{~s}^{-1}$,

where $k_{\mathrm{B}}$ is the Boltzmann constant and $T_{\mathrm{sh}}=8 \times 10^{5} \mathrm{~K}$.

From the Rankine-Hugoniot shock conditions (e.g. Landau \& Lifshits 1987) we know that

$\frac{\rho_{2}}{\rho_{1}}=\frac{(\gamma+1)}{\frac{2}{M^{2}}+(\gamma-1)}$,

where $\rho_{2}$ and $\rho_{1}$ are the post-shock and pre-shock plasma mass densities, $\gamma$ is the ratio of the specific heats $(\gamma=1$ for an isothermal plasma), and $M$ is the Mach number:

$M=\frac{v_{1}}{c_{\mathrm{s}}}$ 

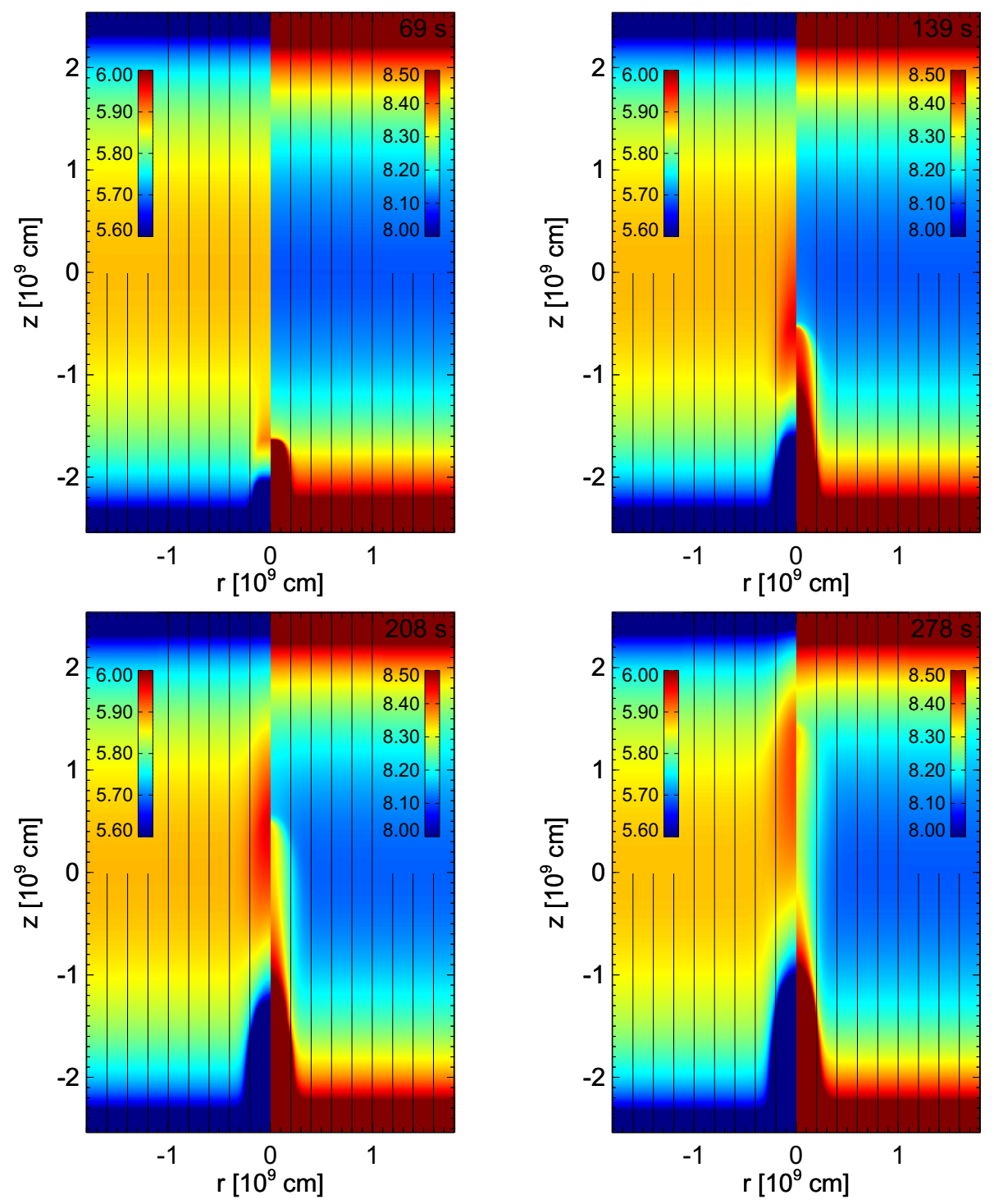

Fig. 3. Results of the reference 2D MHD simulation: maps of temperature (left side) and density (right side) in a cross-section of the cylindrical domain at the labelled times $(t=$ 69 s, 139 s, 208 s, 278 s, see online supplementary movie for an animated version of this evolution).

where $v_{1}$ is the pre-shock plasma speed in the reference frame of the shock front. We measure $v_{1} \approx 140 \mathrm{~km} \mathrm{~s}^{-1}$, that is, $M \approx 1.4$. For $\gamma=1$, we obtain $\rho_{2} / \rho_{1} \approx 2$, which is fully compatible with the density jump in Fig. 5.

Moreover, again from Rankine-Hugoniot shock conditions we have $v_{2}=\left(\rho_{1} / \rho_{2}\right) v_{1} \approx 0.5 v_{1} \approx 70 \mathrm{~km} \mathrm{~s}^{-1}$ in the shock frame and $140-70=70 \mathrm{~km} \mathrm{~s}^{-1}$ in the rest frame. This agrees well with the peak velocity shown in Fig. 5. The propagation of the jet and shock alters the loop equilibrium. At $t=278 \mathrm{~s}$, the shock has travelled all along the loop. At the same time, the length of the coronal part of the loop has effectively decreased because one low part of it is now occupied by the chromospheric jet. We estimate that the loop half-length decreases from $L \sim 2.5 \times 10^{9} \mathrm{~cm}$ to $\sim 1.8 \times 10^{9} \mathrm{~cm}$. According to the loop scaling laws (Rosner et al. 1978), we would expect that at equilibrium and with no change of steady heating, a loop shortening corresponds to (somewhat less than linear) reductions of the pressure and temperature. Instead, because of the shock, at $t=278 \mathrm{~s}$, the plasma pressure has increased from $\sim 0.03 \mathrm{dyn} \mathrm{cm}^{-2}$ to $\sim 0.04 \mathrm{dyn}^{-2}$, and the temperature from $\sim 0.7 \mathrm{MK}$ to $\sim 0.9 \mathrm{MK}$. Therefore, if we interpret this result in terms of equilibrium conditions, we might say that a conversion of kinetic into thermal energy associated with the shock corresponds to an additional heating input into the loop.
For different jet speeds, the solutions are qualitatively very similar; we only obtain slower or faster shock propagation (of about a few tens of seconds), and a smaller or higher density jump at the shock $( \pm 10-20 \%)$ for slower or faster jet speeds, respectively. For a higher density of the jet, the shock moves at the same speed as for the lower density, but the shock jump is about $20 \%$ higher in density.

In Fig. 6 we show a snapshot of the evolution that we obtained in the simulation with a higher value of plasma $\beta$. Although the confinement is no longer as efficient and the cold jet spreads out in the corona, the propagation of the shock ahead of the jet is little affected and remains very similar to that obtained for low $\beta$.

Finally, we also comment on the possible jet propagation in an initially hotter loop (1.5 MK). A shock front still propagates along the loop, with a higher speed but quite a low density jump ( 30\%).

\subsection{Synthetic emission}

Our interest here is mainly in the implications of this jet propagation for emissions in the EUV band. The jet itself is too cold to expect emission in this band. Instead, the shocked plasma is at coronal temperatures, therefore we expect that the density excess 

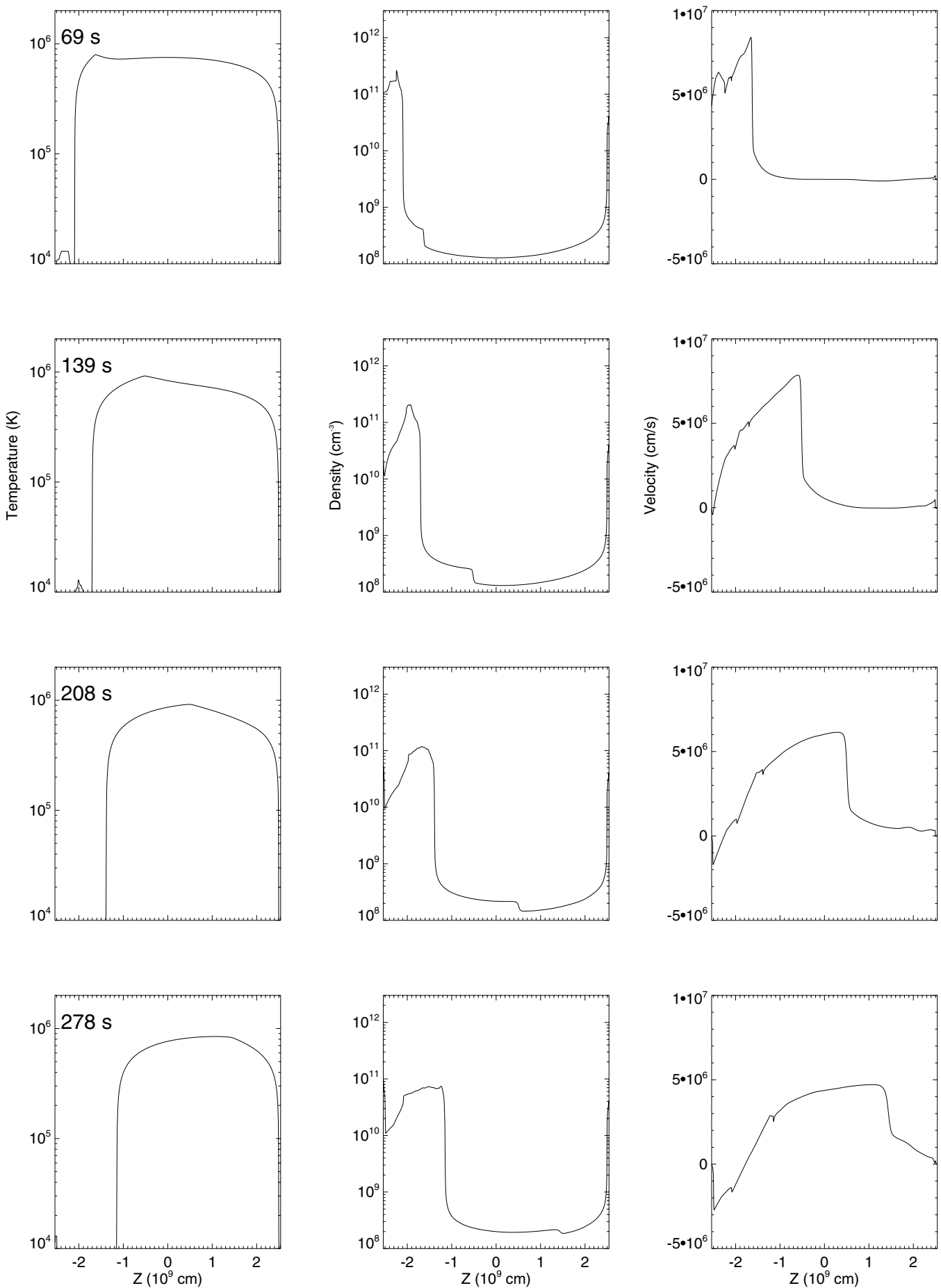

Fig. 4. Results of the MHD simulation: temperature (left), density (middle), and velocity (right) profiles along the central axis of the cylindrical domain at the labelled times.

of the post-shock plasma leads to an emission excess in the EUV band with respect to the unperturbed atmosphere. In other words, a bright shock wave that propagates along the loop might be detectable in the EUV band.

To test this expectation we synthesised the emission in the $171 \AA$ channel of the AIA instrument (Lemen et al. 2012) on board the Solar Dynamics Observatory (SDO) mission. The filterband of this channel includes a very intense Fe IX line with a temperature of maximum formation of $\sim 10^{6} \mathrm{~K}$. The emission in the selected channel is calculated as

$I_{171}(r, z, t)=G_{171}[T(r, z, t)] \operatorname{EM}(r, z, t)$, where

$E M(r, z, t)=n_{e}^{2}(r, z, t) A_{\mathrm{pix}}$.

EM is the emission measure, $G_{171}$ is the sensitivity of the channel as a function of the temperature of the emitting plasma (available from the SolarSoftware package), and the AIA pixel area $A_{\text {pix }}$ is assumed. The depth along the line of sight is left as a free parameter. We computed $I_{171}(r, z, t)$ for each grid cell of our $2 \mathrm{D}$ geometric domain, that is, in the area across the central axis.

Figures 7 and 8 show four maps of the emission detectable in the $171 \AA$ channel at the same times and in a domain 

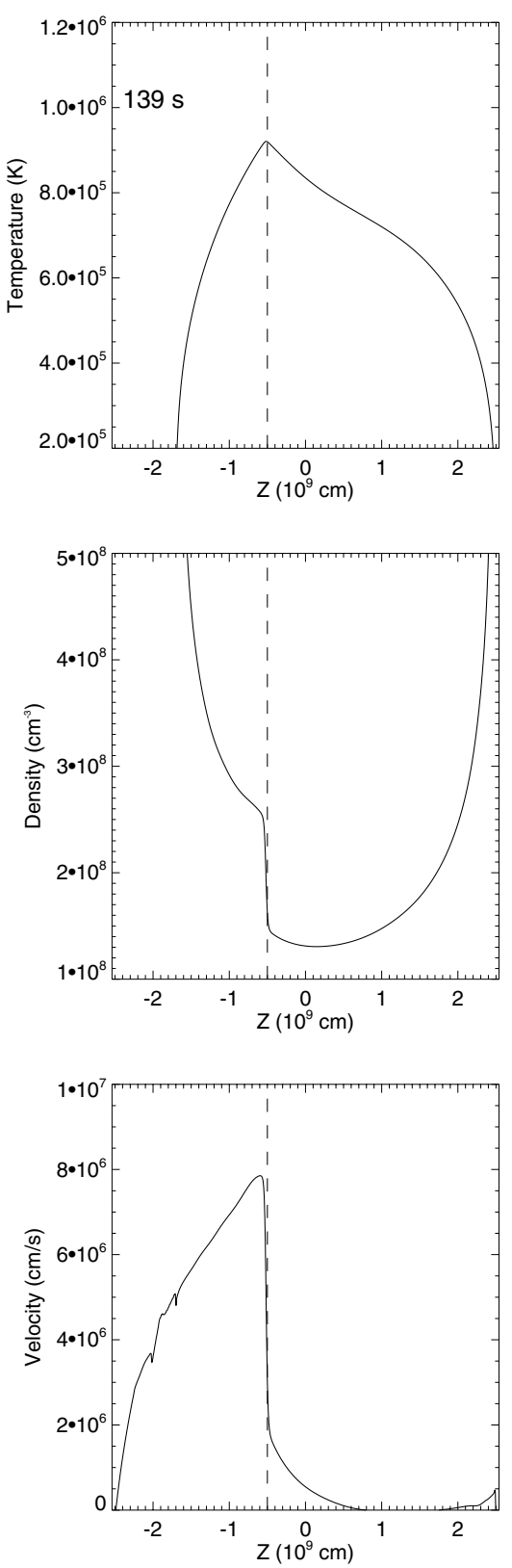

Fig. 5. Detail of Fig. 4 at time $t=139 \mathrm{~s}$ (temperature, density, and velocity). The position of the shock front is marked (vertical dashed line).

cross-section as Fig. 3, and the related profiles along the cen$\operatorname{tral} z$ axis for the reference model.

In Fig. 7 we clearly see the brightening driven by the shock propagation. The bright front propagates along the loop. This brightening is more intense at the beginning and then progressively fades as the shock moves along the coronal loop. The cold jet is the slower black bump. At $t=278 \mathrm{~s}$ we see a faint distinct halo at the opposite edge, which marks the presence of the weak thermal front. This effect would hardly be detected in the observations. In Fig. 8 , initially the emission of the post-shock medium is higher by about a factor 5 than the pre-shock medium (the upper part of the loop), but it is only a slight excess at time $t=278 \mathrm{~s}$. This is about 3 min after the jet injection has ceased.

If we consider that the line-of-sight thickness of the bright moving front might be of about $10^{7}-10^{8} \mathrm{~cm}$, we can estimate that the expected count rate is of about $1-10 \mathrm{DN} \mathrm{s}^{-1} \mathrm{pix}^{-1}$, which

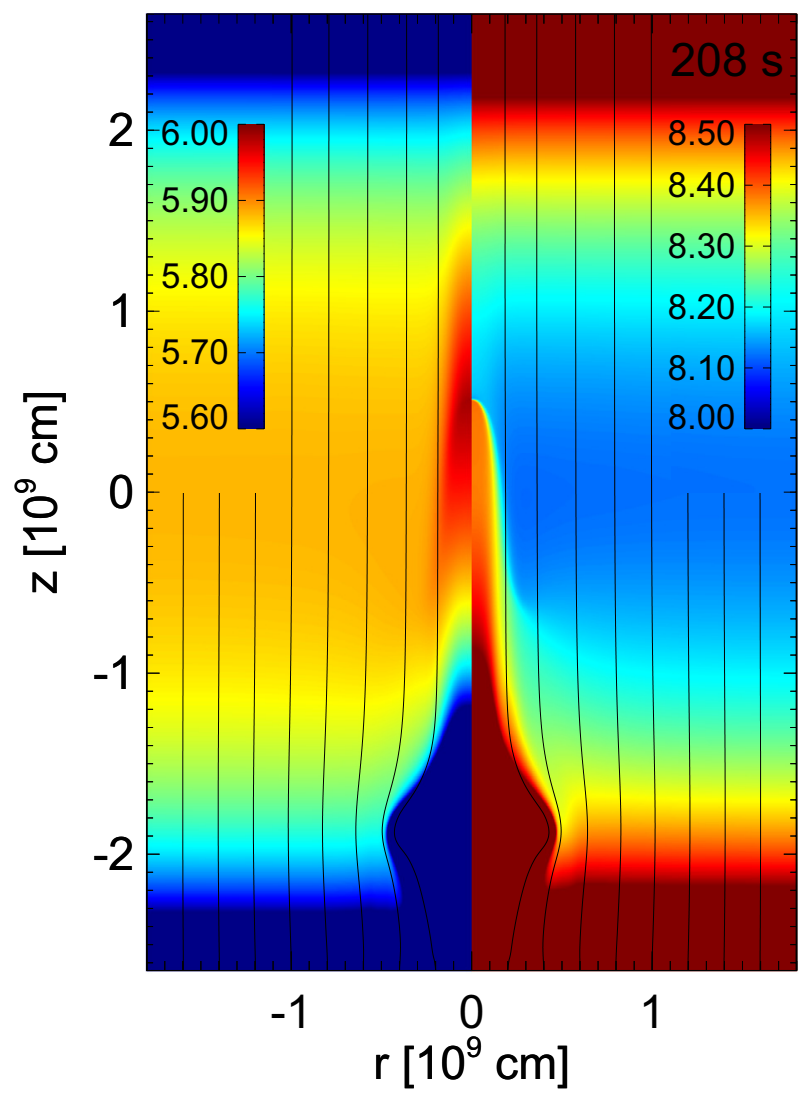

Fig. 6. High $\beta$ MHD simulation: as in Fig. 3 at time $t=208 \mathrm{~s}$ (see online supplementary movie for an animated version of this evolution).

can be measured in actual AIA observations. We remark that the apparent speed of the moving (shock) front is about $140 \mathrm{~km} \mathrm{~s}^{-1}$, while the actual post-shock speed (measurable from Doppler shifts) would be slower than $100 \mathrm{~km} \mathrm{~s}^{-1}$. In the other simulations, we only see some quantitative differences in the shock speed and front brightness, higher for higher jet speed and density. The hotter loop is already EUV bright since the beginning, and the shock perturbs the emission for a very short time and to a much lower extent, thus making the effect of the propagation much less observable.

\section{Discussion and conclusions}

We investigated chromospheric flows ejected upward into a coronal loop. We considered a flow at a speed compatible with that measured for the so-called type II spicules. The aim was to address their observed spatial and temporal correspondence with local brightenings in the extreme-ultraviolet band, emitted by plasma typically at coronal temperatures. We considered a model of a simplified magnetic flux tube, considering a uniform magnetic field and a complete solar atmosphere from the chromosphere to the corona, and including all the physical terms of interest, in particular, gravity, radiative losses, thermal conduction along the field lines, and magnetic induction. The model solved numerically the MHD equations in 2D cylindrical geometry, implemented in the PLUTO parallel code. The chromospheric flow was set at $10^{4} \mathrm{~K}$ and ejected upward at a speed of $30-90 \mathrm{~km} \mathrm{~s}^{-1}$, causing the formation of a shock that preceded it. We considered a realistic jet duration of about 1 min. The stream did not have sufficient initial impulse to reach the apex of the loop, while the shock front swept the entire loop, resulting in a compression of the coronal loop plasma where it 

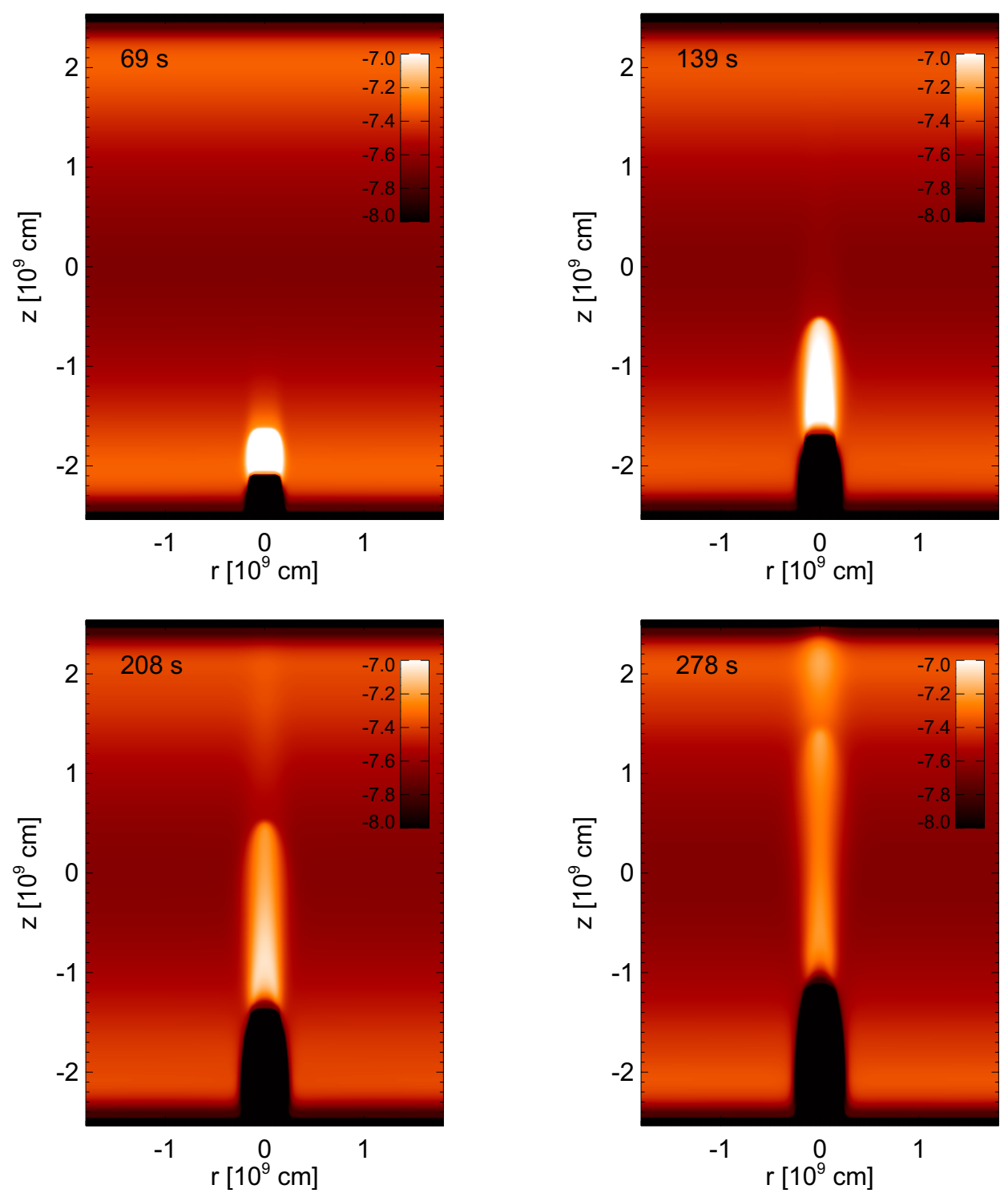

Fig. 7. Maps of synthesized emission (DN $\mathrm{cm}^{-1} \mathrm{~s}^{-1} \mathrm{pix}^{-1}$ ) in the $171 \AA$ channel of the AIA instrument on board of the SDO mission. We show a section of the computational domain at the same times as Fig. 3 (see online supplementary movie for an animated version of this evolution).

propagates. The temperature of post-shock plasma increased to about $1 \times 10^{6} \mathrm{~K}$ and the density by a factor two. Both of these factors led to a substantial increase of the plasma emission, just in the EUV band around $171 \AA$, where the correspondence with type II spicules is observed. This therefore suggests that at least part of the observed evidence can be produced by the formation of these shock fronts after chromospheric flows.

Our model assumptions probably do not affect the generality of this result. Our highly efficient thermal conduction, while typical of low corona conditions, can only lead to underestimate the predicted brightness of the post-shock plasma. Less efficient conduction would approach adiabatic conditions and therefore increase the density jump (up to a factor 4) at the shock front. We do not expect significant differences of the jet evolution for different jet widths. Neither we do not expect large differences in the presence of significant loop expansion in the transition region, because the shock would equally propagate in a corona with a constant cross-section. At most, the absolute intensity might increase because the thickness of the emitting region would increase along the line of sight. We also tested that different jet speed and density only cause some quantitative differences, thus making the overall result robust in the observed ranges.
We emphasize that while this mechanism might explain EUV observations of spicules, it does not explain the corona at large. Our model begins with a corona that is maintained at $T \sim 8 \times 10^{5} \mathrm{~K}$ by steady coronal heating that is unrelated to the spicule. The primary effect of the shock is to compress the pre-existing hot plasma so that it becomes brighter. The shock increases the temperature only slightly to approximately $10^{6} \mathrm{~K}$. Note that the density, emission measure, and therefore brightness of the compressed plasma depend on the initial density in the loop. If the initial plasma were less dense because of a weaker coronal heating rate, there would be less material accumulating ahead of the jet, and the speed of the shock front would be reduced (closer to that of the jet). The sound speed of the initial equilibrium would also be slower, but only very slightly so, since it varies as $n^{1 / 4}$ (using the loop scaling laws in Rosner et al. 1978). Consequently, the Mach number and therefore the shock compression ratio would be smaller, and the EUV emission would be fainter. On the other hand, modelling the jet propagation inside a loop that is already hot $(\sim 1.5 \mathrm{MK})$ since the beginning shows much weaker visible effects.

We note that the scenario studied here is different from that in Klimchuk (2012). Our jet is not subjected to any extra heating, while Klimchuk considered a case where the tip of the 
A. Petralia et al.: MHD modelling of coronal loops
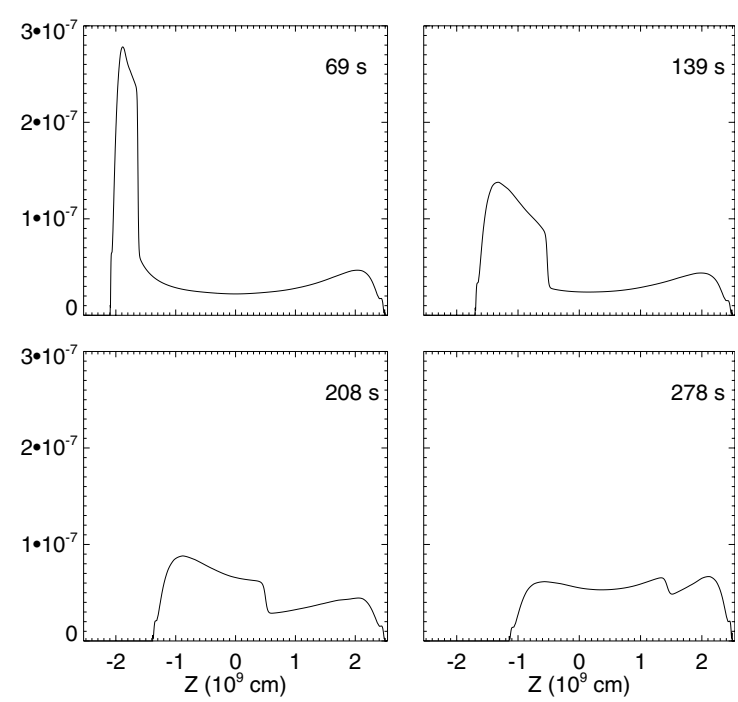

Fig. 8. Emission profiles along the central axis of the cylindrical domain at the same times as Fig. 7.

dense spicule itself was heated to coronal temperatures during the ejection.

In conclusion, the study presented here proposes that shocks might be able to explain the EUV emission in connection with type II spicules. From a broader perspective, this certainly leads the way to a series of other investigations of dynamic flows observed on the Sun in great detail by the SDO mission and others such as the Hinode mission. The MHD model developed here can be applied to a wide variety of flows and their interaction with magnetic fields. These studies may lead to new knowledge that can even go beyond solar physics, as was recently demonstrated with the connection between solar free-falling flows and the accretion flows of star formation (Reale et al. 2013).

Acknowledgements. The authors thank the anonymous referee for suggestions. A.P., F.R., and S.O. acknowledge support from the Italian Ministero dell'Università e Ricerca. The work of J.A.K. was supported by the NASA Supporting Research and Technology Program. PLUTO is developed at the Turin Astronomical Observatory in collaboration with the Department of Physics of Turin University. We acknowledge the HPC facility (SCAN) of the INAF - Osservatorio Astronomico di Palermo, for the availability of high performance computing resources and support. CHIANTI is a collaborative project involving the NRL (USA), the Universities of Florence (Italy) and Cambridge (UK), and George Mason University (USA).

\section{References}

Alexiades, V., Amiez, G., \& Gremaud, P.-A. 1996, Comm. Numer. Methods Eng., 12, 31

Anders, E., \& Grevesse, N. 1989, Geochim. Cosmochim. Acta, 53, 197

Athay, R. G., \& Holzer, T. E. 1982, ApJ, 255, 743

Balsara, D. S., \& Spicer, D. S. 1999, J. Comput. Phys., 149, 270

Beckers, J. M. 1978, ApJ, 224, L143

Bradshaw, S. J., \& Cargill, P. J. 2013, ApJ, 770, 12

De Pontieu, B., McIntosh, S., Hansteen, V. H., et al. 2007, PASJ, 59, 655

De Pontieu, B., McIntosh, S. W., Hansteen, V. H., \& Schrijver, C. J. 2009, ApJ, 701, L1

De Pontieu, B., McIntosh, S. W., Carlsson, M., et al. 2011, Science, 331, 55

Dere, K. P. 2009, A\&A, 497, 287

Giuliani, Jr., J. L. 1984, ApJ, 277, 605

Guarrasi, M., Reale, F., Orlando, S., Mignone, A., \& Klimchuk, J. A. 2014, A\&A, 564, A48

Karpen, J. T., Oran, E. S., Mariska, J. T., Boris, J. P., \& Brueckner, G. E. 1982, ApJ, 261, 375

Klimchuk, J. A. 2012, J. Geophys. Res. (Space Physics), 117, 12102

Landau, L. D., \& Lifshits, E. M. 1987, Fluid mechanics (Oxford, England; New York: Pergamon Press)

Landi, E., Del Zanna, G., Young, P. R., Dere, K. P., \& Mason, H. E. 2012, ApJ, 744, 99

Lemen, J. R., Title, A. M., Akin, D. J., et al. 2012, Sol. Phys., 275, 17

Madjarska, M. S., Vanninathan, K., \& Doyle, J. G. 2011, A\&A, 532, L1

Mignone, A., Bodo, G., Massaglia, S., et al. 2007, ApJS, 170, 228

Mignone, A., Zanni, C., Tzeferacos, P., et al. 2012, ApJS, 198, 7

Orlando, S., Peres, G., Reale, F., et al. 2005, A\&A, 444, 505

Orlando, S., Bocchino, F., Reale, F., Peres, G., \& Pagano, P. 2008, ApJ, 678, 274

Patsourakos, S., Klimchuk, J. A., \& Young, P. R. 2014, ApJ, 781, 58

Pneuman, G. W., \& Kopp, R. A. 1978, Sol. Phys., 57, 49

Reale, F. 2010, Liv. Rev. Sol. Phys., 7, 5

Reale, F., Orlando, S., Testa, P., et al. 2013, Science, 341, 251

Rosner, R., Tucker, W. H., \& Vaiana, G. S. 1978, ApJ, 220, 643

Tripathi, D., \& Klimchuk, J. A. 2013, ApJ, 779, 1

Tsiropoula, G., \& Tziotziou, K. 2004, A\&A, 424, 279

Vanninathan, K., Madjarska, M. S., Scullion, E., \& Doyle, J. G. 2012, Sol. Phys., 280,425 